Surface Science 77 (1978) 505-512

(c) North-Holland Publishing Company

\title{
RELATIVISTIC EFFECTS ON LEED INTENSITIES FROM Au(111)
}

\author{
R. FEDER \\ Institut für Festkörperforschung, KFA, Postfach 1913, D-5170 Jülich 1, Germany \\ and \\ W. MORITZ \\ Institut für Kristallographie, Universität München, Theresienstrasse 41, \\ D-8000 München 2, Germany
}

Received 18 May 1978

Comparison of relativistically and nonrelativistically calculated intensity versus energy profiles in low energy electron diffraction (LEED) from the (111) surface of $\mathrm{Au}(Z=79)$ reveals that relativistic corrections are quite significant. They can however, be obtained in very good approximation by quasirelativistic calculations, in which spin-averaged relativistic phase shifts are used as input for the nonrelativistic multiple scattering formalism. Further, relativistic effects on intensities are found to be comparable to differences arising from different approximations to the exchange part of the ion core potential.

\section{Introduction}

Low energy electron diffraction (LEED) has established itself as one of the most important techniques for studying structural properties of crystal surfaces [1]. The theoretical intensities, which are required for a LEED structure analysis, are usually obtained by solving the Schrödinger equation for an effective crystal potential model, i.e. the assumption is made that a nonrelativistic treatment is adequate for the low energies $(<250 \mathrm{eV}$ ) involved. For heavy (large $Z$ ) materials, relativistic corrections become, however, important: firstly, the diffracted beams show strong spin polarization effects, which were predicted by theory $[2,3]$ and observed experimentally [4,5] and secondly, relativistically calculated intensities for W(110) [3], $\mathrm{W}(001)$ [6] and $\mathrm{Pt}(111)$ [7] were found to deviate significantly from their nonrelativistically calculated counterparts and to yield better agreement with the experiment. With recent interest focusing on the structure of heavy transition metal surfaces, it is therefore pertinent to study relativistic effects on LEED intensities more extensively and to establish their importance for structure determination.

In the present paper, we compare theoretical intensity versus energy profiles for the (111) surface of $\mathrm{Au}$, which were calculated - ceteris paribus - using the Dirac 
equation on the one hand, the Schrödinger equation on the other. Au(111) was chosen for the following reasons. Firstly, the large atomic number of $\mathrm{Au}(Z=79)$ suggests that relativistic effects should be particular strong. Secondly, an fcc (111) surface offers rapid convergence of the computations with respect to the number of beams and layers. Thirdly, intensity measurements were in progress on an $\mathrm{Au}(111)$ surface, which seemed to be unreconstructed. Since details of the crystal potential such as the exchange-correlation approximation can also affect the intensity profiles $[8-10]$ we have studied their influence as well and compare it with the influence of relativistic corrections.

In section 2, we discuss relativistic effects and outline our methods and model assumptions. In section 3 we present and discuss numerical results.

\section{Theory}

The widely used muffin tin approximation, which assumes the one electron crystal potential as spherically symmetric around the ion cores and as constant in the interstitial region, permits the calculation of LEED intensities to proceed in two separate steps: firstly the scattering by a single muffin tin atom and secondly the multiple scattering between the atoms. As a consequence of the Coulomb singularity, relativistic effects are important in the first step, i.e. the single atom scattering has to be described by the Dirac equation, the integration of which yields spin-up and spin-down shifts $\delta_{l}^{*}$ and $\delta_{l}^{-}$. Although the full Dirac equation is required for quantitative results [11] and used in our numerical work, qualitative insight into relativistic effects can already be obtained from a two-component approximation to the order $1 / c^{2}$, the correction terms in which are [12(a)]

$-\frac{p^{4}}{8 m^{3} c^{2}}+\frac{\hbar^{2}}{8 m^{2} c^{2}} \Delta V(r)+\frac{\hbar^{2}}{4 m^{2} c^{2}} \frac{\mathrm{d} V(r)}{\mathrm{d} r}(\sigma \cdot L)$,

where $p$ is the momentum operator, $V(r)$ the muffin tin potential, $\boldsymbol{\sigma}$ the Pauli spin operator and $L$ the angular momentum operator. While the third term, which describes spin-orbit coupling, produces a spin-splitting of nonrelativistic phase shifts $\tilde{\delta}_{\text {}}$, the first two terms (relativistic mass correction and Darwin term) only shift the $\delta_{l}$. The sign of this shift can be deduced from an integral representation of the phase shifts in terms of the scattering potentials [12b]. Both terms being negative, we expect, for a given energy, the relativistic phase shifts to be displaced by a positive amount with respect to the phase shifts $\widetilde{\delta}_{l}$ obtained by means of the Schrödinger equation.

For a description of the nonrelativistic and the relativistic multiple scattering formalisms used for our calculations, we refer to earlier work [13-15]. Since convergence of the results with respect to the number of sites in the lattice summation, the number of beams and layers is ensured, differences between relativistic and non- 
relativistic LEED intensities can be attributed to relativistic effects (already apparent in the phase shifts), provided that the physical model assumptions are the same for both programs. These assumptions involve in particular a crystal model, which is simply a truncation of the bulk (i.e. no surface reconstruction) an inner potential with real part $0[16]$ and imaginary part $4 \mathrm{eV}$. The crystal temperature was taken as $0 \mathrm{~K}$.

As for the muffin potential, we use - for the study of relativistic effects - a relativistic band structure potential [17], in which exchange and correlation effects were taken care of in the customary Slater approximation. Since recent model calculations have shown that LEED intensities can be rather sensitive to the potential approximation [8-10], it is of interest to compare the magnitude of relativistic effects with potential effects. To this end. we have carried out further nonrelativistic calculations using potentials, in which the exchange interaction is treated in the Hartree-Fock approximation [18] and in a local density approximation [19].

Since the relativistic multiple scattering computer program requires about four times as much storage space and about 7 times as much execution time as the nonrelativistic program, it seems worthwhile to investigate the accuracy of the following quasirelativistic approximation. The average values
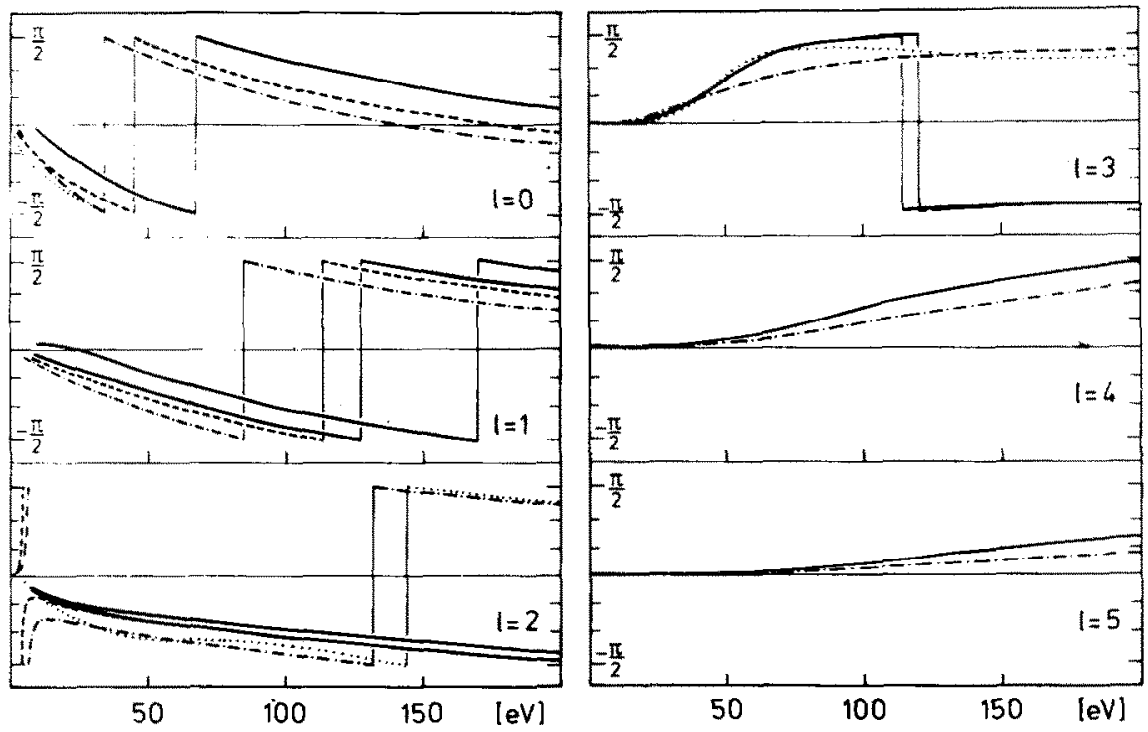

Fig. 1. Phase shifts for Au. (-) Relativistic $\delta_{l}^{+}$and $\delta_{\bar{l}}$ from band structure potential. $(---)$ Nonrelativistic from band structure potential. (-.-.) Nonrelativistic from atomic potential with Slater-Wilson-Wood exchange. $(\cdots \cdots)$ Nonrelativistic from atomic potential with Hartree-Fock-Pendry exchange. (For $l>2$, the nonrelativistic band structure phase shifts coincide with the relativistic ones, and for $l>3$, the phase shifts for the two energydependent exchange approximations are also practically the same.) 


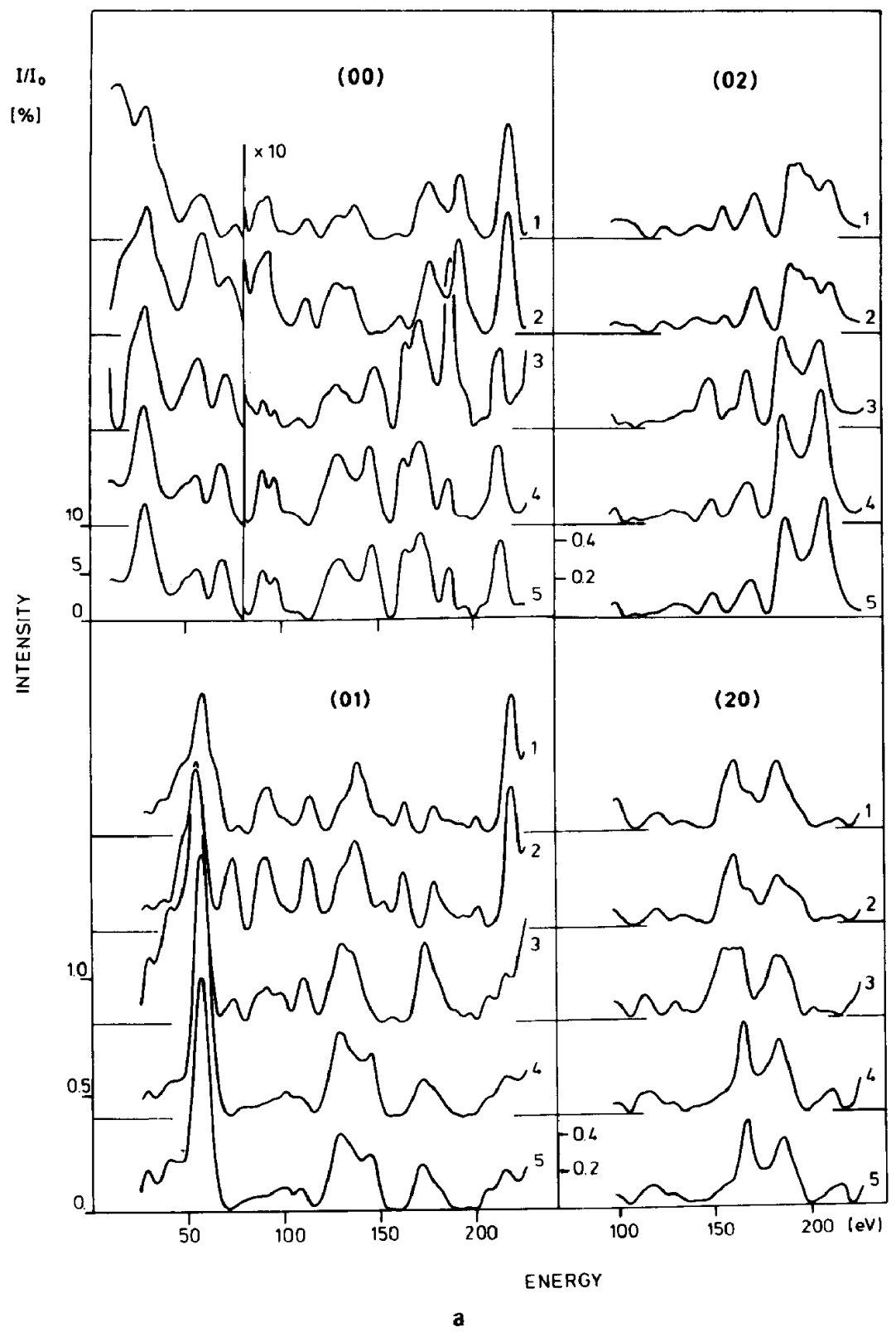




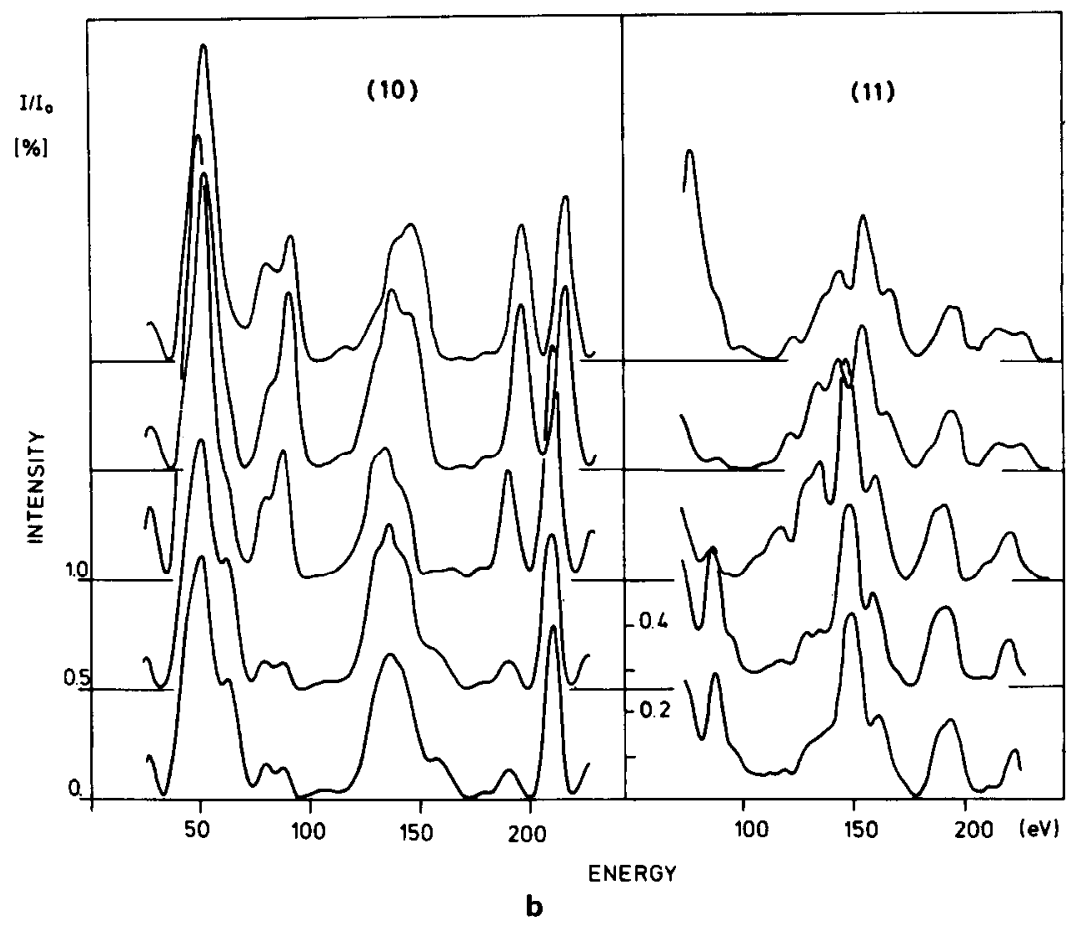

Fig. 2. Theoretical intensity versus energy profiles of LEED beams (as indicated in each panel) for normal incidence on $\mathrm{Au}(111)$ at $T=0 \mathrm{~K}$. Eight phase shifts were used. The calculation was (in each panel): (1) nonrelativistic with SWW potential. (2) nonrelativistic with HEP potential, (3) nonrelativistic with band structure potential, (4) quasirelativistic with band structure potential, (5) relativistic with band structural potential.

$\delta_{l}=\left(\delta_{l}^{+}+\delta_{l}^{-}\right) / 2$

of the proper relativistic phase shifts are used as input for the nonrelativistic multiple scattering program [20].

\section{Numerical results}

Ion core scattering phase shifts up to $l=7$ were obtained by numerical integration of the Dirac and the Schrödinger equation and are shown - up to $l=5-$ in fig. 1. Comparing the relativistic and nonrelativistic phase shifts from the band structure potential, we notice that the former are raised against the latter, as was anticipated above, the difference being largest for $l=0,1$ and decreasing with increase $l$. The spin-orbit splitting, which is absent in our nonrelativistic approximation, 
is seen to be considerable only for $l=1$ and negligible as from $l=3$. As for the nonrelativistic phase shifts obtained from the other two potentials that contain an energy dependent exchange contribution, we find significantly lower values than for the band structure potential phase shifts for all $l$. This is plausible on the grounds of the integral representation of the phase shifts (cf. above), since the band structure potential involves the Slater exchange approximation, which - at energies above the Fermi level - overestimates exchange effects and thereby lowers the potential. The Hartree-Fock phase shifts and the Slater-Wilson-Wood phase shifts differ from each other mainly below about $100 \mathrm{eV}$. Such behaviour with energy is to be expected, since the exchange interaction between a free electron of energy $E$ and bound electrons decreases with increasing $E$.

In fig. 2 we present intensity versus energy profiles of several LEED beams from $\mathrm{Au}(111)$ at normal incidence, which were obtained by the methods described above using the various sets of phase shifts. For the band structure potential, the nonrelativistic profiles show peaks essentially at the same energy positions as the relativistic profiles, but relative peak intensities as well as peak shapes are significantly different. The quasirelativistic approximation is seen to yield intensity profiles almost identical to the proper relativistic ones for all the beams. Since the relativistic and quasirelativistic phase shifts differ mainly by the comparatively small spin-orbit splitting of $\delta_{1}$, which implies very similar ion core scattering cross sections, it thus appears that coherent multiple scattering does not enhance this difference.

Comparing the nonrelativistic intensity profiles obtained for the different exchange approximations (curves 1, 2, 3 in fig. 2), we notice significant discrepancies. In the energy range above $100 \mathrm{eV}$, where exchange is in fact almost negligible, the two energy dependent exchange approximations produce almost identical intensities (curves 1 and 2), which differ from the constant exchange approximation results (curve 3 ) not only with respect to peak shapes and relative heights but also with respect to peak positions, the peaks of 1 and 2 being generally shifted by about $7 \mathrm{eV}$ towards higher energies against the peaks of 3 . Such a shift can be qualitatively understood as a consequence of the excess negative potential energy (effective inner potential) in the constant exchange approximation. Below about $100 \mathrm{eV}$ curves 1 and 2 also differ significantly from each other.

The question, whether exchange effects or relativistic effects are "stronger", can only be readily answered when specialized to individual features of individual beams. For example, the height ratio of the peaks near 55 and $70 \mathrm{eV}$ in the (00) profile or that of the peaks near 80 and $90 \mathrm{eV}$ in the (10) profile is far more strongly influenced by relativity. So is a peak near $90 \mathrm{eV}$ in the (01) profile, which is present in all nonrelativistic results and absent in the relativistic ones. On the other hand, there are features, like the (01) peak near $170 \mathrm{eV}$, for which exchange effects dominate, It thus appears that relativistic effects and exchange effects are of comparable importance.

Comparison of our results with experimental intensity profiles [21] show good overall agreement. Since the LEED pattern indicates a slight reconstruction of the 
surface [21-23], a detailed comparison between our calculations, which assume a perfectly periodic unreconstructed surface, and the available experimental data, does, however, not seem meaningful, unless one can identify the changes induced by the reconstruction.

\section{Conclusion}

Our calculations show that relativistic effects on LEED intensity profiles from $\mathrm{Au}(111)$ are significant. They are much larger than the marginal differences between theoretical and experimental results, which are acceptable in present-day LEED structure analysis (cf. ref. [1]). and must therefore definitely be taken into account. This can be achieved in very good approximation without additional computational labour by using a nonrelativistic multiple scattering program with a spin averaged relativistic phase shift input [24]. We note that our findings are in accordance with earlier work on W(001), and expect them to hold quite generally for surfaces of heavy materials (i.e. atomic number larger than about 50). Discrepancies between the LEED intensities obtained for different approximations to the exchange potential are found to be comparable to the relativistic corrections, which implies that a LEED structure analysis using the above quasirelativistic method still requires care in choosing the ion core potential model (cf. refs. [8-10]).

\section{Acknowledgements}

One of us (W.M.) is grateful for financial support by the Deutsche Forschungsgemeinschaft (SFB 128).

\section{References}

[1] F. Jona and P.M. Marcus, Comments Solid State Phys. 8 (1977) 1.

[2] P.J. Jennings, Japan. J. Appl. Phys. Suppl. 2, Pt. 2 (1974) 661.

[3] R. Feder, Phys. Status Solidi (b) 62 (1974) 135.

[4] M.R. O'Neill, M. Kalisvaart, F.B. Dunning and G.K. Walters, Phys. Rev. Letters 34 (1975) 1167 ;

M. Kalisvaart, M.R. O'Neill, T.W. Riddle, F.B. Dunning and G.K. Walters, Phys. Rev. B17 (1978) 1570.

[5] N. Müller and D. Wolf, Bull. Am. Phys. Soc. II, 21 (1976) 944;

R. Feder, N. Müller and D. Wolf, Z. Physik B28 (1977) 265.

[6] P.J. Jennings and M.N. Read, J. Phys. C8 (1975) L285.

[7] R. Feder, Surface Sci. 68 (1977) 229.

[8] S.Y. Tong, J.B. Pendry and L.L. Kesmodel, Surface Sci. 54 (1976) 21.

[9] P.M. Echenique, J. Phys. C9 (1976) 3193.

[10] P.M. Echenique and D.J. Titterington, J. Phys. C10 (1977) 625;

F. Soria, J.L. Sacedon, P.M. Echenique and D.J. Titterington, Surface Sci. 68 (1977) 448. 
[11] H.J. Meister and H.F. Weiss, Z. Physik 216 (1968) 165.

[12] A. Messiah, Quantum Mechanics (North-Holland, Amsterdam, 1965) (a) Vol. II, ch. X, section 35; (b) Vol. I, ch. X, section 17.

[13] J.B. Pendry, Low Energy Electron Diffraction (Academic Press, New York, 1974).

[14] R. Feder, Phys. Rev. Letters 36 (1976) 598.

[15] R. Feder, P.J. Jennings and R.O. Jones, Surface Sci. 61 (1976) 307

[16] This is justified for normal incidence calculations, if the surface barrier is assumed as nonreflecting.

[17] O.K. Andersen, private communication.

[18] This approximation, while found less adequate for Ni (cf. ref. [8]), was successfully used for the noble metals Cu (J.B. Pendry, J. Phys. C4 (1971) 2514) and Ag (W. Moritz, Ph.D. thesis, Munich (1976)).

[19] J.C. Slater, T.M. Wilson and J.W. Wood, Phys. Rev. 179 (1969) 28.

[20] This approximation was found to be reasonable by R. Feder and J.B. Pendry (1972, unpublished) and P.J. Jennings and M.N. Read (J. Phys. C8 (1975) L285) for W(001) over a rather small range of low energies.

[21] A. Zimmer, to be published.

[22] D. Wolf, Ph.D. thesis, Munich (1972).

[23] D.M. Zehner and J.F. Wendelken, in: Proc. 7 th Intern. Vacuum Congr. and 3rd Intern. Conf. on Solid Surfaces, Vienna, 1977.

[24] Whilst our calculations were done only for normal incidence, our conclusions are also valid for off-normal incidence by virtue of the reciprocity theorem beased on time reversal symmetry. 